\title{
Evaluation of Dynamic Intimal Flap Movement Using IVUS in Patients with Type B Aortic Dissections Before and after TEVAR
}

\author{
Halim Yammine*, Jocelyn K Ballast, Joel Harding, Jeko M Madjarov, Tzvi Nussbaum and Frank R Arko \\ Sanger Heart and Vascular Institute, Atrium Health, 1001 Blythe Blvd Ste 300, Charlotte, NC 28203, USA \\ *Corresponding author: Halim Yammine, 1001 Blythe Blvd Ste 300, Charlotte, NC 28203, USA
}

\author{
ARTICLE INFO \\ Received: 幽 January 28, 2019 \\ Published: 㓞 February 07, 2019
}

Citation: Halim Y, Jocelyn K B, Joel H Jeko M M, Tzvi N, Frank R A. Evaluation of Dynamic Intimal Flap Movement Using IVUS in Patients with Type B Aortic Dissections Before and after TEVAR. Biomed J Sci \& Tech Res 14(2)2019. BJSTR. MS.ID.002520.

Keywords: TEVAR; IVUS; Type B Aortic Dissection; Intimal Flap; False Lumen; True Lumen; Stent Graft

Abbreviations: TBAD: Type B Aortic Dissections; IVUS: Intravascular Ultrasound; FMI: Flap Movement Index; FAI: Flap Area Index; AD: Aortic Diameters; TD: True Lumen Diameters; TEVAR: Thoracic Endovascular Aortic Repair

\section{ABSTRACT}

Objective: Evaluation and characterization of intimal flaps within the visceral aorta before and after thoracic endovascular stent graft placement in Stanford type B aortic dissections (TBAD).

Methods: We evaluated 19 patients with TBAD: 11 chronic and 8 acute (less than 14 days). Intravascular Ultrasound (IVUS) recorded aortic flap movement at the level of the superior mesenteric artery during one RR-interval of the ECG. Flap movement was record before and after stent-graft placement. We defined and evaluated the following; intimal flap as the flap movement index (FMI) and flap area index (FAI) with measurements of the minimum and maximum aortic diameters (ADmin, ADmax), minimum and maximum true lumen diameters (TDmin, TDmax), as well as true lumen areas (TAmin, TAmax). Flap movement index was defined as [(TDmax/ADmax-TDmin/ADmin)]/TDmin/ADmin x 100 (\%). The flap area index was defined as TAmax-TAmin/TAmin x100. Results are reported as the mean $+/$ - the standard deviation. Significant $P$ values are less than 0.05 .

Results: Mortality and SCI rates were $0 \%$. All patients were successfully treated with complete thrombosis of the false lumen of the thoracic aorta at 1-month follow-up as shown by CTA. There was nearly complete remodeling of the thoracic aorta. There was an immediate and significant increase in the true lumen diameters and areas following stent graft repair in the visceral aorta. Following thoracic stent graft repair, the flap movement index and flap area index were reduced from $19.63 \pm 2.3 \%$ to $10.66 \pm 1.9 \%(\mathrm{P}=.0001)$ and $95.65 \pm 21 \%$ to $18.85 \pm 4.2 \%(\mathrm{P}=.0001)$, respectively.

Conclusion: Thoracic aortic stent grafting has become the treatment of choice for patients with complicated TBAD. This is the first study to evaluate and characterize the dynamic aortic flap movement of the visceral aorta in patients with TBAD immediately before and after thoracic stent graft repair using IVUS.

\section{Introduction}

Since its initial use in treating TBAD was reported in 1999, thoracic endovascular aortic repair (TEVAR) has evolved as the treatment of choice for complicated descending thoracic aortic dissections [1-6]. Endovascular repair in TBAD attempts to reestablish the pre-dissection hemodynamics of the aorta by covering the proximal entry tear and stimulating aortic remodeling [7]. Numerous studies have demonstrated aortic remodeling after TEVAR for TBAD $[8,9]$. Aortic remodeling, characterized by expansion of the true lumen and thrombosis and healing of the false lumen, is associated with the best outcomes following TEVAR [8,1012].

The immediate effects of TEVAR, however, are less frequently described. In an aortic dissection, the septum is of varying thickness and mobility. It is also sensitive to changes in the hemodynamic pressures within the aorta, often contributing to dynamic stenosis or occlusion of branch vessels. The placement of a thoracic stent 
graft has the potential to immediately improve flow within the true lumen and reduce dynamic obstruction caused by the intimal flap. The purpose of this study was to describe the intimal flap movement at the level of the visceral aorta intra-operatively prior to and following the deployment of the endograft in patients with TBAD.

\section{Methods}

Between Sept 2011 and Aug 2012, 19 consecutive patients were treated with thoracic stent grafts for type B aortic dissections. IVUS examination prior to stent graft placement was performed from the ascending aorta to the external iliac arteries. An 8.2F, 8.35MHz Visions catheter (Volcano Corp., Rancho Cordova, CA, USA) was introduced over a 0.035 inch stiff wire through a $10 \mathrm{~cm}$ $8 \mathrm{~F}$ sheath and advanced to the ascending aorta. The IVUS catheter was oriented when the renal vein was identified to correctly adjust the image. With the gain set at 40 to more accurately delineate the aortic flap and lumen, a 10 second video loop of aortic and aortic flap motion was recorded within the visceral aorta at the SMA level during RR-intervals of the ECG prior to and after the deployment of the stent graft.

\section{Analysis of IVUS Data}

The video recordings of the aortic and aortic flap movement were played on a DICOM viewer (Rubo Medical, Aerdenhout, The Netherlands) to evaluate the movement throughout a single cardiac cycle. A Scion PCI Frame Grabber (Scion Corporation, Frederick, MD, USA) was used to capture still images, which were then analyzed using Image J software to measure diameters and luminal areas. The minimum (at diastole) and maximum (at systole) total aortic lumen diameter and area as well as the minimum and maximum true lumen diameter and area were measured. Measurements were obtained from the inner wall to the inner wall, the lumen was then bounded and the total areas of the aorta and of the true lumen were assessed quantitatively in square centimeters. Each movement was independently reviewed by two blinded observers to evaluate the diameter and area change of the aorta and the true lumen. Measurements were taken of minimum and maximum aortic diameters (ADmin, ADmax), minimum and maximum true lumen diameters (TDmin, TDmax), and true lumen areas (TAmin, TAmax). Flap movement index (FMI), a description of the amount of movement of the intimal flap over the course of a single cardiac cycle, was defined as [(TDmax/ADmax-TDmin/ADmin)/(TDmin/ ADmin $x$ 100)]. Flap area index (FAI), a description of change in true lumen area over the course of a single cardiac cycle, was defined as [(TAmax-TAmin)/TAmin x 100].

\section{Statistical Analysis}

Measures of diameter and area are described as mean \pm standard deviation. Difference in area and diameters from systole to diastole were evaluated using a Student t-test for paired data. Differences in measurements before and after TEVAR were evaluated using analysis of variance for repeated measurements. Analyses of measurement method comparison data according to Bland and Altman were performed to analyze repeatability and to compare measurements by two observers (there was no significant difference between the measurements of the two observers at any point during this study). A P value less than 0.05 was considered statistically significant.

\section{Results}

Of the 19 patients with TBAD treated with TEVAR, 11 were chronic ( $>2$ weeks), and 8 acute $(<2$ weeks). All patients were treated with the Valiant thoracic stent graft. Coverage in all patients was proximally from zone 2 (coverage of the left subclavian artery), with a distal extent down to $2 \mathrm{~cm}$ above the celiac artery. All procedures were considered successful, and 1 month followup CTA demonstrated complete thrombosis of the false lumen with nearly complete remodeling within the thoracic aorta. There were no instances of mortality or spinal cord ischemia within 30 days postoperatively. Table 1 demonstrates the measurements seen on IVUS. The placement of a thoracic stent graft did not cause significant change in the total aortic diameter (AD), either at systole (ADmax) or at diastole (ADmin).

Table 1: IVUS measurements of total aortic diameter and true lumen area and diameter at systole (max) and diastole (min) before and after placement of thoracic stent to treat TBAD.

\begin{tabular}{|c|c|c|c|}
\hline & Pre Thoracic Stent & $\begin{array}{c}\text { Post Thoracic } \\
\text { Stent }\end{array}$ & P value \\
\hline TD min $(\mathrm{mm})^{*}$ & $11.25 \pm 1.7$ & $16.00 \pm 1.8$ & 0.0001 \\
\hline TD max $(\mathrm{mm}) \dagger$ & $14.50 \pm 1.29$ & $19.25 \pm 2.2$ & 0.0001 \\
\hline TA min $(\mathrm{cm} 2) \ddagger$ & $1.38 \pm 0.21$ & $2.97 \pm 0.33$ & 0.0001 \\
\hline TA max $(\mathrm{cm} 2) \S$ & $2.70 \pm 0.42$ & $3.53 \pm 0.22$ & 0.0001 \\
\hline AD $\min (\mathrm{mm})[]$ & $22.75 \pm 1.5$ & $22.75 \pm 1.5$ & 0.9 \\
\hline AD max $(\mathrm{mm}) \|$ & $24.5 \pm 2.08$ & $24.75 \pm 2.2$ & 0.8 \\
\hline
\end{tabular}

* TD min: minimum true lumen diameter

† TD max: maximum true lumen diameter

$\ddagger$ TA min: minimum true lumen area

$\S$ TA max: maximum true lumen area

[] AD min: minimum aortic diameter

\section{| | AD max: maximum aortic diameter}

Prior to intervention, the true lumen diameter (TD) changed significantly throughout the cardiac cycle, with the true lumen diameter changing from $11.25 \pm 1.7 \mathrm{~mm}$ in diastole (TDmin) to $14.50 \pm 1.29 \mathrm{~mm}$ in systole (TDmax) (P=.01). Following TEVAR, true lumen diameter was significantly larger than before at both systole and diastole $(\mathrm{P}=.001)$. It also continued to change significantly during the cardiac cycle (TDmin $16.00 \pm 1.8 \mathrm{~mm}$ vs TD max $19.25 \pm 2.2 \mathrm{~mm} \mathrm{P=.01)}$ ). Prior to TEVAR, the true lumen area (TA) changed significantly during the cardiac cycle between diastole (TAmin) and systole (TAmax) $\left(1.38 \pm 0.21 \mathrm{~cm}^{2}\right.$ vs $2.70 \pm 0.42 \mathrm{~cm} 2$ 
respectively $\mathrm{P}=.0001)$. After TEVAR, the true lumen area became significantly larger. In addition, it continued to change significantly during the cardiac cycle (TAmin $2.97 \pm 0.33 \mathrm{~cm} 2$ vs TAmax 3.53 $\pm 0.22 \mathrm{~cm} 2 \mathrm{P}=.0001$ ). Following thoracic stent graft repair, FMI was significantly decreased from $19.63 \pm 2.3 \%$ to $10.66 \pm 1.9 \%$ $(\mathrm{P}=.0001)$. In addition, FAI was also significantly decreased from $95.65 \pm 21 \%$ to $18.85 \pm 4.2 \%(\mathrm{P}=.0001)$.

\section{Discussion}

Since 1999, when the first papers describing the use of endografts to treat complicated type B dissections were published, TEVAR has been found to be a safe and effective alternative to open repair. It has been also shown to provide improved 5-year outcomes when compared to optimal medical therapy alone [1$3,6]$. The primary goal of TEVAR during treatment of TBAD is to exclude blood flow to the false lumen. Persistent flow within the false lumen, especially partial thrombosis of the false lumen, is an important risk factor for aortic enlargement, and false lumen thrombosis is associated with improved outcomes [13-16]. TEVAR promotes false lumen thrombosis and remodeling by covering the primary entry tear and expanding the true lumen.

TEVAR can significantly alter the morphology of the true and false lumen $[8,17]$. Schoder et al. followed changes in the TL and FL diameter in 20 patients after TEVAR for acute complicated dissection with $90 \%$ achievement of FL thrombosis at one year with a mean decrease in the FL diameter of $11.6 \mathrm{~mm}$ [15]. Conrad et al also documented aortic remodeling in 21 patients in the year following TEVAR, 88\% of whom had thrombosis of the FL [10]. The mean maximum diameter of the TL in Conrad's study significantly increased from 23.7 to $31.1 \mathrm{~mm}$ and the mean maximum aortic diameter decreased from 39.9 to $34.8 \mathrm{~mm}$ [10]. In our study, $100 \%$ of patients experienced false lumen thrombosis within 30 days after TEVAR, and we found that there was a significant increase in the size of the true lumen in all patients. Similarly, we found a significant increase in the volume of the true lumen after TEVAR. In a volumetric analysis study, Stanley et al. showed that false lumen thrombosis after TEVAR was associated with a significant increase in the volume of the true lumen as a percentage of the total aortic volume from $42.7 \%$ to $61.7 \%$.

On the other hand, continued false lumen patency did not affect the true lumen volume percentage after TEVAR (46.7\% to $47.7 \%$ ) [18]. It is our practice to maximize the coverage of the thoracic aorta in patients with TBAD, since remodeling may not occur in segments that are not stented [19]. In this study, we evaluated the visceral segment of the aorta, which often remains untreated and has fenestrations present, allowing continued flow into the false lumen. In this study, we were able to clearly demonstrate that with maximum thoracic stent graft coverage to just above the celiac artery, there is a significant increase in the true lumen diameter and volume even in the untreated visceral segment of the aorta. Compression of the true lumen can be dramatic, especially in acute dissections, significantly reducing flow through distal branches perfused by the normal flow channel. TEVAR has the potential to stabilize the often highly mobile septum, which is sensitive to changes in the hemodynamic pressures within the aorta and can cause dynamic stenosis or occlusion of branch vessels.

Our data suggest that not only does TEVAR promote longterm aortic remodeling, it provides immediate stabilization of the intimal flap and increase in true lumen diameter and volume at the visceral segment, reducing the time that patients may experience reduced blood-flow due to true lumen compression, be it static or dynamic. Unlike other studies which have utilized postoperative CTA to assess aortic remodeling, the current study assessed aortic diameter, true lumen diameter, and true lumen area intraoperatively, immediately after insertion of the stent-graft. IVUS clearly demonstrated that there is a significant increase in true lumen diameter and volume immediately after TEVAR. We were also able to dynamically study the aortic intimal flap with the use of IVUS and to assess the immediate effects of intervention on flap mobility. We found a significant decrease in the movement (i.e. stabilization of the intimal flap) immediately after maximum coverage of the thoracic aorta.

\section{Conclusions}

Thoracic aortic stent grafting is becoming the standard of care in patients with complicated TBAD. Aortic morphologic changes occur immediately after stent graft placement with a significant increase in the true lumen diameter and area. Furthermore, the stent graft significantly minimizes the movement of the intimal flap. Further studies to determine the clinical significance of flap stabilization are required, however, the use of intravascular ultrasound to measure the flap intimal index and area may be a useful adjunct to predict false lumen thrombosis.

\section{References}

1. Dake MD, Kato N, Mitchell RS, Semba CP, Razavi MK, et al. (1999) Endovascular stent-graft placement for treatment of acute aortic dissection. N Engl J Med 340(20): 1546-1552.

2. Nienaber CA, Fattori R, Lund G, Dieckmann C, Wolf W, et al. (1999) Nonsurgical reconstruction of thoracic aortic dissection by stent-graft placement. N Engl J Med 340(20): 1539-1545.

3. Fattori R, Montgomery D, Lovato L, Kische S, DiEusanio M, et al. (2013) Survival after endovascular therapy in patients with type B aortic dissection: a report from the International Registry of Acute Aortic Dissection (IRAD). JACC Cardiovasc Interv 6(8): 876-882.

4. Hagan PG, Nienaber CA, Isselbacher EM, et al. (2000) The International Registry of Acute Aortic Dissection (IRAD). JAMA 283(7): 897-903.

5. Clouse WD, Hallet JW, Schaff HV, Spittell PC, Rowland CM, et al. (2004) Acute aortic dissection: population-based incidence compared with degenerative aortic aneurysm rupture. Mayo Clin Proc 79(2): 176-180.

6. Nienaber CA, Kische S, Rousseau H, Rehders TC, Kundt G, et al. (2014) Endovascular Repair of Type B Aortic Dissection: Long-term Results of the Randomized Investigation of Stent Grafts in Aortic Dissection Trial. J Vasc Surg 6(4): 407-416.

7. Akutsu K, Nejima J, Kiuchi K, Sasaki K, Ochi M, et al. (2004) Effects of the patent false lumen on the long-term outcome of type B aortic dissection. Eur J Cardiothoracic Surg 26(2): 359-366. 
8. Huptas S, Mehta RH, Kühl H, Tsagakis K, Reinsch N, et al. (2009) Aortic remodeling in type $\mathrm{B}$ aortic dissection: effects of endovascular stentgraft repair and medical treatment on true and false lumen volumes. J Endovasc Ther 16(1): 28-38.

9. Patterson BO, Cobb RJ, Karthikesalingam A, Holt PJ, Hinchliffe RJ, et al. (2014) A systematic review of aortic remodeling after endovascular repair of type B aortic dissection: methods and outcomes. Ann Thorac Surg 97(2): 588-595.

10. Conrad MF, Crawford RS, Kwolek CJ, Brewster DC, Brady TJ, et al. (2009) Aortic remodeling after endovascular repair of acute complicated type B aortic dissection. J Vasc Surg 50(3): 510-517.

11. Durham CA, Aranson NJ, Ergul EA, Wang LJ, Patel VI, et al. (2015) Aneurysmal degeneration of the thoracoabdominal aorta after medical management of type B aortic dissections. J Vasc Surg 62(4): 900-906.

12. Watanabe Y, Shimamura K, Yoshida T, Daimon T, Shirakawa Y, et al (2014) Aortic remodeling as a prognostic factor for late aortic events after thoracic endovascular aortic repair in type B aortic dissection with patent false lumen. J Endovasc Ther 21(4): 517-525.

13. Sueyoshi E, Sakamoto I, Hayashi K, Yamaguchi T, Imada T (2004) Growth rate of aortic diameter in patients with type $\mathrm{B}$ aortic dissection during the chronic phase. Circulation 110(11 suppl 1): 256-261.

\section{ISSN: 2574-1241}

DOI: 10.26717.BJSTR.2019.14.002520

Halim Yammine. Biomed J Sci \& Tech Res

(C) This work is licensed under Creative

Submission Link: https://biomedres.us/submit-manuscript.php
14. Trimarchi S, Tolenaar JL, Jonker FH, Murray B, Tsai TT, et al. (2013) Importance of false lumen thrombosis in type B aortic dissection prognosis. J Thorac Cardiovasc Surg 145(3): S208-S212.

15. Schoder M, Czerny M, Cejna M, Rand T, Stadler A, et al. (2007) Endovascular repair of acute type B aortic dissection: long-term followup of true and false lumen diameter changes. Ann Thorac Surg 83(3): 1059-1066.

16. Tolenaar JL, Kern JA, Jonker FH, Cherry KJ, Tracci MC, et al. (2014) Predictors of false lumen thrombosis in type B aortic dissection treated with TEVAR. Annals of cardiothoracic surgery 3(3): 255-263.

17. Kim KM, Donayre CE, Reynolds TS, Kopchok GE, Walot I, et al. (2011) Aortic remodeling, volumetric analysis, and clinical outcomes of endoluminal exclusion of acute complicated type B thoracic aortic dissections. J Vasc Surg 54(2): 316-325.

18. Stanley GA, Murphy EH, Knowles M, Ilves M, Jessen ME, et al. (2011) Volumetric analysis of type B aortic dissections treated with thoracic endovascular aortic repair. J Vasc Surg 54(4): 985-992.

19. Kang WC, Greenberg RK, Mastracci TM, Eagleton MJ, Hernandez AV, et al. (2011) Endovascular repair of complicated chronic distal aortic dissections: intermediate outcomes and complications. J Thorac Cardiovasc Surg 142(5): 1074-1083.

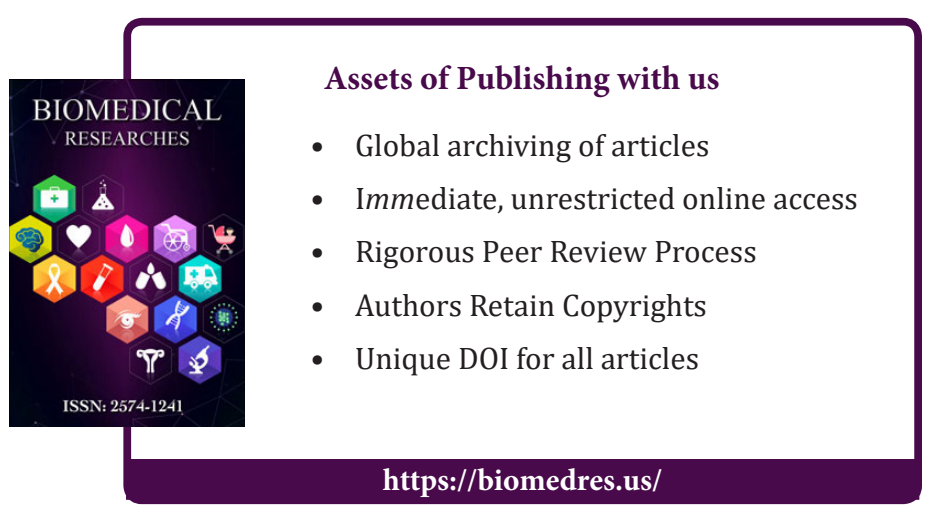

